\title{
“NO NOS SENTIMOS IDENTIFICADAS". LA ÉTICA DE LO CONCRETO EN LOS PROCESOS DE INVESTIGACIÓN CON PERSONAS MIGRANTES
}

\author{
"We do not feel ourselves identified". The ethics of the concrete \\ in research processes with migrants
}

Rosalía López Fernández*

\begin{abstract}
Resumen. A partir del análisis de una experiencia de investigación realizada con mujeres migrantes, este texto se orienta a poner a de manifiesto una serie de cuestiones de carácter ético que acontecieron en el proceso de investigación y en la posterior difusión de sus resultados. En concreto se abordarán las implicaciones que tiene para la investigación la "reificación" de los sujetos migrantes y su conceptualización como "objetos" de estudio, así como el uso de determinadas categorías para su representación. Igualmente se considerarán toda una serie de cuestiones epistemológicas que en los estudios sobre migraciones redundan en una violencia simbólica fruto de la relación asimétrica entre investigadores y sujetos etnificados. A modo de reflexión final, se expondrá la necesidad de disponer de un corpus de principios éticos que pueda dar respuesta a las particularidades de la investigación desarrollada en el ámbito de los estudios migratorios.
\end{abstract}

Palabras clave: ética, mujeres, migración, investigación, epistemología.

\begin{abstract}
Based on the analysis of a research experience with migrant women, this text aims at revealing a series of ethical issues that occurred while the research process and the subsequent dissemination of its results. Specifically, I will examine the implications that reification of migrant subjects and their conceptualization as "objects" of study, as well as the use of certain categories for their representation. I will also consider a whole series of epistemological issues that in migration studies may finish as a symbolic violence resulting from the asymmetric relationship between researchers and ethnicized subjects. As a final reflection, I will expose the need to set out a corpus of ethical principles that may be able to respond to the particularities of the research carried out in the field of migration studies.
\end{abstract}

Keywords: ethics, women, migration, research, epistemology.

\footnotetext{
* Instituto de Migraciones, Universidad de Granada. Granada, España.
} 


\section{Introducción}

Como señala Márquez Fernández ${ }^{1}$ el conocimiento no es neutro, como tampoco lo es la investigación científica de la que surge. Menos aún lo son los sujetos que dan origen a tal actividad. Es por esto que la producción de conocimiento se entiende como un acto necesariamente situado e intencional y como un proceso en el que los investigadores deben avanzar tomando decisiones de carácter ético. Así, en cualquier área de conocimiento y en cualquier disciplina científica, se requiere de una serie de principios éticos, más o menos explícitos, que garanticen, entre otras cuestiones, "la credibilidad de ese conocimiento y su aplicación humanitaria"².

Quizás el aspecto más importante dentro de la ética de la investigación tiene que ver con todas aquellas consideraciones que afectan a los sujetos junto con los que se realizan las investigaciones, por lo que "la forma como se trata a las personas y los beneficios, aunque sean indirectos, que puedan obtener de su participación son cuestiones críticas que es preciso mantener en primer término" ${ }^{3}$. En este orden de ideas, la seguridad, la dignidad y la integridad de los participantes deben ser elementos irrenunciables e independientes de los objetivos de la investigación y sus resultados.

En el campo concreto de las migraciones los marcos éticos desde los que sostener las prácticas investigadoras deberían tener en cuenta la naturaleza misma de los fenómenos migratorios, así como la variedad de objetos teóricos que pueden abordarse en su estudio. Si consideramos a las migraciones, desde la noción propuesta por Marcel Mauss, como "hechos sociales totales" ${ }^{4}$, estos marcos éticos deberían poder adecuarse a todos los elementos de la estructura social y a todo tipo de instituciones en tanto que no existe fenómeno ni grupo social que no se pueda asociar al estudio de las migraciones.

La investigación en migraciones debería partir, por tanto, de aquellos aspectos éticos comunes y aplicables a cualquier tipo de investigación de carácter social, aunque el análisis de cuestiones pertenecientes al ámbito de los estudios migratorios podría requerir de análisis éticos más específicos. La realidad concreta, como punto de partida, puede estimular la reflexión en torno a ciertos aspectos problemáticos de la investigación en migraciones y sobre los cuales no se están articulando debates de naturaleza ética.

Con el fin de poder profundizar en algunos aspectos concretos sobre dilemas éticos en los estudios migratorios, las reflexiones que a continuación se presentan,

1 MÁRQUEZ FERNÁNDEZ, Álvaro. Ética del investigador frente a la producción y difusión del conocimiento científico, p. 640.

2 Ibidem, p. 640.

3 MESÍA MARAVÍ, Rubén. Contexto ético de la investigación social, p. 139.

4 PRADA, Miguel Ángel de, ACTIS, Walter, PEREDA, Carlos. ¿Cómo abordar el estudio de las migraciones? Propuesta teórico metodológica, p. 51. 
se refieren a un estudio de caso y surgen tras la escritura de una comunicación titulada "Luchas y aprendizajes desde espacios subalternos en el contexto asociativo. Procesos de empoderamiento y participación de mujeres migrantes empleadas de hogar" que iba a presentarse a un Congreso. El punto central fue que el contenido del texto no fue "aprobado" por el equipo coordinador de la asociación de mujeres migrantes cuya experiencia se estaba narrando. Las cuestiones sobre las que se quiere reflexionar en este texto constituyen una crítica a las decisiones tomadas para la difusión del texto, así como al contenido del mismo en sus aspectos ontológicos, epistemológicos y conceptuales. Estas reflexiones surgen también como legado de transparencia y de compromiso que como investigadores debemos mantener con la comunidad científica, en la que es poco frecuente encontrar experiencias donde se hagan visibles los errores y las dificultades que se producen en el transcurso de las investigaciones.

\section{Unas breves notas sobre el contenido del texto}

El texto de la comunicación sobre la que se pretende problematizar, se escribió tras un periodo de participación observante ${ }^{5}$ de ocho meses de duración en el que la asociación de mujeres que hemos mencionado, inicialmente formada por mujeres procedentes de un único país, había iniciado un proceso de reflexión colectivo sobre si debían convertirse en una asociación a la que pudieran acceder personas procedentes de cualquier país y si se enfocaba su actividad específicamente en el empleo de hogar y a los cuidados. Esta participación observante fue completada con distintas entrevistas tanto a personas que estaban encargadas de la coordinación de la asociación, como a personas que participaban en las actividades que se estaban realizando ${ }^{6}$.

\subsection{Objetivo, marco teórico y análisis del texto}

Cuando surge la posibilidad de escribir un texto para presentar un análisis sobre el proceso que se había llevado a cabo y sobre el significado del mismo en un congreso científico, se contactó con el equipo coordinador de la asociación informando sobre este hecho al que dieron el visto bueno para enviar un resumen

5 La participación observante se distingue de la observación participante por el grado de implicación en las tareas y actividades que se desarrollan en la asociación, así como un tipo de observación que, por ser implicado, no pierde capacidad de análisis crítico de la realidad y rigor en el procedimiento. Para más información ver: BECKER, Howard, GEER, Blanche. Participant observation: the analysis of qualitative field data; y KAWULICH, Barbara. Participant Observation as a Data Collection Method.

6 He de señalar que mi rol en la asociación era doble y explícito, pues, por un lado, era un miembro más siendo mi función la de documentar el proceso que se estaba siguiendo y de registrar los aprendizajes, los errores, ya que uno de los hándicaps de determinadas asociaciones es que no cuentan con agentes que hagan relatos que permitan conservar la memoria colectiva del repertorio de acciones realizadas, de modo que los procesos, las movilizaciones y los aprendizajes se van perdiendo. Pero, por otro, era sabido que me encontraba realizando una investigación sobre representaciones de pobreza de mujeres migrantes empleadas de hogar. 
que pudiera ser evaluado. Dicho resumen fue aprobado por los organizadores del simposio al que se remitió por lo que el siguiente paso fue escribir una comunicación de "carácter académico" cuyo objetivo así se detallaba:

El objetivo del presente texto es el de analizar la trayectoria de la asociación, y en especial de los procesos de empoderamiento llevados a cabo por este grupo de mujeres, que merece especial interés puesto que se trata de la primera asociación de mujeres inmigrantes por el empleo de hogar en esta ciudad. La forma asociativa está posibilitando una nueva forma de interlocución frente a otras organizaciones que se dedican al empleo de hogar, así como frente a instituciones públicas y partidos políticos. Esto supone la destradicionalización de las dinámicas reivindicativas en este contexto ya que anteriormente han sido otros colectivos, no encabezados por mujeres, los que han realizado las labores de lucha y de exigencia de los derechos de las personas empleadas de hogar?.

El marco teórico elegido para el texto partía de la necesidad de deconstruir críticamente los discursos sociales y académicos hegemónicos que sobre los migrantes y las migraciones se han ido elaborando y que los representan como meros objetos pasivos de estudio a merced de los condicionantes culturales, sociales, económicos y políticos de las sociedades de destino que los identifican como sujetos incompetentes e incapaces de modelar el desarrollo de su proyecto migratorio. Con respecto a las mujeres migrantes, se pretendía señalar la constante invisibilización a la que han sido sometidas en la literatura académica occidental y cómo sus saberes han sido ignorados y desestimados en pos de un conocimiento de carácter científico. Por todo esto, la Sociología de las Ausencias y de las Emergencias posibilitaba articular estas cuestiones.

En este texto, realizado desde una perspectiva feminista de género, partiré de la Sociología de las Ausencias en tanto que esta centra su atención en aquellas experiencias sociales que están siendo desperdiciadas, olvidadas, ignoradas o reprimidas. La racionalidad occidental ha privilegiado ciertos tipos de saberes relegando al ostracismo a otras formas de hacer y de producir conocimiento y esto supone la "dilapidación de una riqueza cultural que debería estar al servicio del empoderamiento de los excluidos $^{\prime \prime}$. Desde la Sociología de las Ausencias se investigará sobre las potencialidades, las posibilidades y las alternativas disponibles que ofrece la asociación y tiene como objetivo mostrar que lo que no existe es, de hecho, activamente producido como no existente, es decir, como una alternativa no creíble a las opciones hegemónicas ${ }^{9}$. Desde la Sociología de las Emergencias se abre el campo de las experiencias y las alternativas sociales posibles en la medida que "actúa tanto sobre las posibilidades

7 LÓPEZ FERNÁNDEZ, Rosalía. Luchas y aprendizajes desde espacios subalternos en el contexto asociativo. Procesos de empoderamiento y participación de mujeres migrantes empleadas de hogar.

8 VIDAL FERNÁNDEZ, Fernando. Pan y rosas. Fundamentos de exclusión social y empoderamiento, p. 201.

9 SOUSA SANTOS, Boaventura de. Epistemologías del Sur, p. 30. 
(potencialidad) como sobre las capacidades (potencia) ${ }^{\prime 10}$. Este recorrido, permite llegar a la Ecología de Saberes ${ }^{11}$, desde la que se tratará de dar lugar a un nuevo tipo de relación entre el conocimiento científico y otros conocimientos $^{12}$.

El análisis teórico realizado en el texto se centró en las ideas de agencia, empoderamiento, ciudadanía y participación. Con respecto al concepto de agencia, se empleó la perspectiva de Lois $\mathrm{McNay}^{13}$ en la que la capacidad de agencia de los sujetos no consiste en una mera reacción a la dominación, sino que esta se conforma desde la conciencia plena de la capacidad de cambio y desde la autonomía y la reflexividad que proviene de la autoconciencia en relación al otro.

Con respecto al empoderamiento femenino, este no podía entenderse sin situarlo dentro del marco de una estructura social, económica, cultural o política concreta que condiciona su propio desarrollo, por lo que podríamos señalar que los procesos de empoderamiento, en este caso, estaban atravesados por la desigualdad de clase. El empoderamiento consistía en

Dentro del ámbito de la asociación el empoderamiento se ha entendido dentro del modelo de donación en el sentido mausiano ${ }^{14}$ que supone corresponsabilidad, solidaridad, redistribución y compromiso social, no solo para con las integrantes de la asociación, sino con cualquier mujer y/o empleada de hogar. Este empoderamiento pasa por la comprensión de los mecanismos de dominación, por la organización y la movilización en los espacios públicos y en la progresiva constitución de una agenda política más amplia ${ }^{15}$.

Estos procesos de empoderamiento se vincularon con el aumento de las posibilidades de la participación de estas mujeres migrantes en los contextos que habitan, pero cuya presencia es una cuestión políticamente irrelevante, culturalmente problemática y estéticamente indeseable. La participación fue enmarcada dentro de la dialéctica de derechos y deberes, haciendo referencia al abuso que se realiza de este discurso cuando se pretende que, por ejemplo, los inmigrantes se integren -o sean asimilados- en las sociedades sin haberles otorgado cierto tipo de derechos. Así, los procesos de integración de la población inmigrante extranjera deberían enmarcarse en el acceso progresivo a una ciudadanía participativa "fundada en concretos derechos y deberes, [que] incluye ciertamente participación en derechos políticos y no solo en derechos

\footnotetext{
10 IDEM. Decolonizar el saber, reinventar el poder, p. 26.

11 IDEM. La Sociología de las Ausencias y la Sociología de las Emergencias: para una ecología de saberes.

12 LÓPEZ FERNÁNDEZ, op. cit.

${ }^{13}$ McNAY, Lois. Gender and Agency: Reconfiguring the Subject in Feminist and Social Theory.

${ }^{14}$ VIDAL FERNÁNDEZ, op. cit., p. 398.

${ }^{15}$ LÓPEZ FERNÁNDEZ, op. cit.
} 
sociales y civiles"16. El concepto de ciudadanía generaba un gran número de contradicciones pues se trata de una condición política de carácter legal que, vinculada con la nacionalidad, hace referencia a un estatus efectivo otorgado por los Estados más que una fidelidad hacia este ${ }^{17}$. En el caso de las personas inmigrantes, la noción de ciudadanía daba lugar a marcadas gradacione ${ }^{18}$ que podrían clasificarla como incompleta o imperfecta. La categoría de ciudadanía estaba, en gran medida, construida a partir de la imagen del varón y desde una estructura de poder patriarcal donde las cualidades y características femeninas estaban devaluadas e intencionalmente desconsideradas. En este tenor, se señalaba la propuesta de Pateman ${ }^{19}$ en tanto que aborda la deconstrucción de la concepción patriarcal, occidentalocéntrica, blanca, capitalocéntrica y cristianocéntrica de ciudadanía que tiene como modelo al varón y que genera a su vez una categoría de extranjeridad no necesariamente vinculada a la nacionalidad, sino más bien a la etnificación de los sujetos migrantes.

\section{2. "No nos sentimos identificadas"}

Esta breve presentación del contenido del texto escrito y de sus fundamentos teóricos será la base para comprender la naturaleza del problema que comenzó cuando, una vez concluido el texto y puesto a disposición de las mujeres con las que había trabajado, me comunicaron que no se sentían identificadas con su contenido y que no me daban la autorización necesaria para enviarlo.

Tras varios intentos de diálogo con el equipo coordinador de la asociación para poder analizar aquellos elementos problemáticos, no fue posible encontrar un espacio conveniente para tratar el contenido, por lo que en esta tesitura consulté a un comité de ética y a distintos investigadores sobre las posibles alternativas para dar salida al texto por dos motivos fundamentales. En primer lugar, y más determinante, porque la experiencia narrada era significativa y podía servir como ejemplo a otros colectivos y, en segundo lugar, porque al tratarse de un congreso científico existía la posibilidad de dialogar con personas expertas en estos temas que pudieran ofrecer nuevas perspectivas y dimensiones sobre las que seguir construyendo pensamiento.

Las recomendaciones recibidas tras las consultas fueron en la línea de enviar el texto al congreso dado que no había información sensible y que, al tratarse de reflexiones de carácter científico, la discusión de su validez y

\footnotetext{
${ }_{16}$ APARICIO GÓMEZ, Rosa, TORNOS CUBILLOS, Andrés. Las asociaciones de inmigrantes en España. Una visión de conjunto, p. 45.

17 SASSEN, Saskia. Contrageografías de la globalización. Género y ciudadanía en los circuitos transfronterizos.

${ }^{18}$ ARANGO VILA-BELDA, Joaquín. Inmigración y diversidad humana. Una nueva era en las migraciones internacionales.

${ }^{19}$ PATEMAN, Carole. The sexual contract.
} 
pertinencia era un debate que debía afrontarse en otros términos y contextos y no en aquellos expresados por las mujeres de la asociación. Previo al envío del texto, debían suprimirse las referencias a personas concretas, los nombres propios, las referencias a lugares, en definitiva, anonimizar el texto de forma que no fuera posible identificar geográfica y personalmente el proceso recogido.

\section{La ética de lo concreto en los procesos de investigación con personas migrantes}

Tras toda esta serie de incidentes y desencuentros sobre los que desafortunadamente no fue posible generar espacios de diálogo, solo quedaba lugar para una reflexión densa y profunda con el fin de analizar qué había pasado y por qué estas mujeres no se habían sentido identificadas con el contenido del texto. Esta reflexión fue desvelando una serie de cuestiones de carácter ético que no fueron tenidas en cuenta durante el proceso de investigación y que, a continuación, serán presentadas en dos apartados complementarios. En primer lugar, se considerarán las implicaciones que tuvo para la investigación la reificación de los sujetos migrantes y su conceptualización como "objetos" de estudio, así como el uso que se hizo de determinadas categorías para su representación. Y, en segundo lugar, se abordarán toda una serie de cuestiones epistemológicas que en los estudios sobre migraciones redundan en una violencia simbólica fruto de la relación asimétrica entre investigadores y sujetos "etnificados".

\subsection{Categorías y conceptos en la investigación "sobre"}

Es de obligada mención comenzar este apartado recordando que mucha de la investigación que se realiza en el área de las migraciones continúa siendo una investigación "sobre" personas migrantes y no "con" personas migrantes. Este hecho tiene como posible base varios argumentos. En primer lugar, podríamos señalar que debido a las relaciones de poder que se generan en la intersección de clase, raza, género y sexualidad, algunas personas migrantes, son ubicadas, de acuerdo con la línea abismal propuesta por Santos, en la zona del no-ser fanoniana y que los confina a una posicionalidad de inferioridad en las relaciones raciale ${ }^{20}$. En segundo lugar, las personas migrantes han sido representadas como "el sumun de la diferencia cultural, el sumun de la otredad"21 para las sociedades que los nombran como "migrantes", que a través de discursos diferencialistas, lógicas de inferiorización y una esencialización de las diferencias culturales, son relegados a una exterioridad categórica que les aparta incluso de su condición de sujetos. Por último, la significación social e histórica con la que se ha ido cargando a los términos "inmigrante" como diferentes o extraños, inferiores

${ }^{20}$ GROSFOGUEL, Ramón. La descolonización del conocimiento: diálogo crítico entre la visión descolonial de Frantz Fanon y la sociología descolonial de Boaventura de Sousa Santos, p. 99.

${ }^{21}$ OLMOS ALCARÁZ, Antonia. La población inmigrante extranjera y la construcción de la diferencia. Discursos de alteridad en el sistema educativo andaluz, p. 111. 
o atrasados y a "inmigración" como una opción miserabilista, amenazante o peligrosa $^{22}$, los convierte en una categoría social pertinente para los científicos sociales y en los objetos de sus investigaciones cuando, como personas, abandonaron las fronteras de sus países y se convirtieron en inmigrantes.

Teniendo presente estas premisas, y volviendo al caso de la investigación que nos ocupa, he de señalar que las relaciones mantenidas durante el trabajo de campo fueron establecidas en la línea de investigar junto "con", aunque en el posterior análisis teórico estas se transformaron en un investigar "sobre". Este cambio, debido principalmente a una falta de vigilancia epistemológica que será abordada más adelante, convertía de facto a estas mujeres en meros objetos sobre los que teorizar y el elenco de experiencias vividas en los datos sobre los que construir el análisis. La investigación que en principio se estableció desde relaciones de igualdad, aunque con roles diferenciados, se transformó, durante el proceso de escritura, en un espacio regido por relaciones de poder y de dominación, en este caso, a través de la descripción ${ }^{23}$ y por la aplicación de ciertos códigos pertenecientes al ámbito de los investigadores.

La imposición de determinadas categorías y conceptos reproduce, sin lugar a dudas, los esquemas presentes en la colonialidad que pasan por formas de dominación que se fundamentan en la superioridad epistémica del investigador. Los lugares epistémicos que yo personalmente pensaba como diferentes, se transformaron, tras la escritura del texto, en posicionamientos jerárquicos de superioridad-inferioridad y como una imposición, al percibir que "mis categorías y conceptos" estaban tratando de explicar su propio mundo sin un punto de consenso.

Entre los investigadores en el área de las migraciones existe la creencia, poco cuestionada, de que las categorías analíticas mediante las cuales se aprehende la realidad son de carácter neutro y anteriores a la propia diversidad de la cual se pretende dejar constancia ${ }^{24}$. Se tiende a pensar que las categorías son "puntos invariables de referencia" que permiten explorar las particularidades de cada grupo humano, dándoles cabida en una red conceptual común y universalmente válida ${ }^{25}$. Esto se debe a que es difícil deconstruir un sistema conceptual hegemónico que parece carecer de alternativas y a que, para muchos

${ }^{22}$ El análisis de las significaciones y el papel que los investigadores sociales han jugado en el uso de determinadas categorías, se encuentra en: SANTAMARÍA LOZANO, Enrique. La incógnita del extraño. Una aproximación a la significación sociológica de la "inmigración comunitaria".

${ }^{23}$ SCHEFFLER, Ted. The ideology of binary opposition: Subject/object Duality and Anthropology, p. 166.

24 Para profundizar sobre el tratamiento que se les da a los sujetos migrantes como objetos de la investigación y sobre la estigmatización que el uso de ciertas categorías tiene en los textos académicos, ver: GARCÍA CASTAÑO, Francisco Javier, LÓPEZ FERNÁNDEZ, Rosalía, THAMM, Miriam. Sujetos y territorios en el estudio de las migraciones desde la antropología en España.

25 SEGATO, Rita Laura. Una paradoja del relativismo. El discurso racional de la antropología frente a lo sagrado, p. 31. 
investigadores, el uso de determinadas categorías se trata de una simple cuestión terminológica y semántica y no un procedimiento creador de realidad ${ }^{26}$. Sin embargo, los actos de nombrar, categorizar, clasificar o conceptualizar poseen una naturaleza eminentemente ética dado el carácter instituyente de estas categorías y las implicaciones y consecuencias que tiene el uso de determinados términos aparentemente neutros. En el caso concreto de este texto, la categoría de "mujeres migrantes empleadas de hogar" que se repetía en numerosas ocasiones, no contenía el mismo matiz político reivindicativo con el que estas mujeres se autodenominaban y se reducía a nombrar a un grupo que, bajo esos términos, podía ser fácilmente percibido como un colectivo socialmente desconsiderado. No menos grave que esta cuestión, fue el hecho de haber equiparado la categoría analítica de "mujeres migrantes empleadas de hogar" a un grupo internamente homogéneo, externamente definido y con unos objetivos compartidos en el marco de la asociación a la que pertenecen. La tendencia a pensar en los colectivos de migrantes como grupos articulados sin que salten las alarmas ante este intento de "grupismo", tiene su base en una concepción etnificada de los sujetos que serviría como criterio amalgamador sin la necesidad de que se compruebe la existencia real de algún tipo de vínculo que justifique la construcción de la categoría "grupo" ${ }^{27}$. La atribución de uniformidad a los colectivos de migrantes es una inferencia frecuente en los estudios sobre migraciones y es síntoma de prácticas de campo superficiales que, sin embargo, pretenden dar cuenta irrebatible de la realidad social que describen.

En esta misma línea, otro de los aspectos problemáticos del texto fue la pérdida total de la literalidad de los discursos que conscientemente se omitió para dar paso a unas explicaciones teóricas acordes a un contexto académico. En estos espacios, la abstracción teórica que ha de hacerse, ha de salir fuera del mundo cognitivo de las personas con las que se trabaja a la vez que sus propias reflexiones son traducidas a otros lenguajes y conceptos. Se abandona así el mundo factual discursivo para situarnos en el plano conceptual analítico que es esencialmente distinto, pero que se ha construido como cualitativamente mejor por tratarse de aquel que le es propio a los investigadores.

El proceso de producción de conocimiento científico desconecta a los sujetos de sus propios saberes a la vez que los deslegitima para su producción por el requerimiento de determinados lenguajes ${ }^{28}$. Esta forma de producir conocimiento expropia a los sujetos la capacidad de definir la realidad en sus propios términos y de resistir las objetivaciones a las que son sometidos por parte de los investigadores ${ }^{29}$. La naturalización de los migrantes como

\footnotetext{
${ }^{26}$ BRUBAKER, Roger, COOPER, Frederik. Beyond "identity", p. 2.

${ }^{27}$ BRUBAKER, Roger. Ethnicity without groups, p. 169.

${ }^{28}$ BERGER, Peter, LUCKMANN, Thomas. La construcción social de la realidad.

${ }^{29}$ VASILACHIS DE GIALDINO, Irene. Pobres, pobreza, identidad y representaciones sociales.
} 
incapaces de dar cuenta de las situaciones que viven es un hecho que la academia se ha encargado de ratificar al representar a las personas migrantes como culturalmente inferiores o como incapaces de dar sentido a sus propias acciones por su condición de "alter", extraño o extranjero ${ }^{30}$.

\subsection{Las exclusiones fueron, en último término, epistemológicas}

Los estudios sobre migraciones, siguiendo la tradición de una ciencia dogmática que representa al conocimiento científicamente producido como aquel aparato privilegiado para la representación del mundo, no han estado ajenos al racismo epistémico ${ }^{31}$ en los que las voces de ciertos sujetos han sido consideradas de segundo orden o directamente han sido silenciadas. Como señala Dipesh Chakrabarty 32 haciendo referencia a la disciplina histórica, "las exclusiones [...], son, en último término, epistemológicas", y esta, sin duda, ha sido una cuestión ética esencial dentro del proceso de investigación que se había llevado a cabo.

Inicialmente, el texto presentado al Congreso partía de la singularidad de la experiencia vivida durante el periodo de conformación de la asociación de mujeres migrantes por el empleo de hogar y los cuidados, así como de las primeras actividades formativas y reivindicativas llevadas a cabo. Todo ello contextualizado en el marco legislativo del empleo doméstico y en la situación de la realidad asociativa de la población migrante. Entre todos los hechos sociales sobre los que podía teorizarse escogí el empoderamiento y la participación, desde la capacidad de agencia y desde la categoría política de ciudadanía. El carácter impositivo de esta forma de proceder convertía a las integrantes de esta asociación en el objeto de la problematización científica que para este texto concreto se había creado. En el caso de la población migrante en general, la problematización intelectual no está, en absoluto, asociada a un problema social, aunque esta dinámica de la práctica investigadora les obliga a vivir de forma casi permanente ligada a "problemas y problemáticas" construidos fuera de su espacio de control. La dominación epistémica de los investigadores también se basa en la posibilidad de construir la relevancia que le es otorgada a una determinada realidad hasta convertirla en un "hecho social" de interés académico, en detrimento de otras posibilidades.

En un segundo momento, cuando se buscó anonimizar el texto, se eliminaron todas las referencias a personas concretas y sus reflexiones, a los lugares y a cualquier otro elemento que hubiera permitido identificar la

\footnotetext{
${ }^{30}$ Para profundizar en las representaciones de los migrantes, ver: HALL, Stuart. El espectáculo del "Otro"; SANTAMARIA LOZANO, Enrique. Inmigración y barbarie. La construcción social y política del inmigrante como amenaza.

${ }^{31}$ GROSFOGUEL, Ramón. La descolonización del conocimiento: diálogo crítico entre la visión decolonial de Frantz Fanon y la sociología descolonial de Boaventura de Sousa Santos, p. 102.

${ }^{32}$ CHAKRABARTY, Dipesh. Historias de las minorías, pasados subalternos, p. 91.
} 
autoría del conocimiento que estas mujeres estaban produciendo, se originó la deshumanización del relato y con ello la negación de la existencia de "otros sujetos" y de otras epistemologías. En este acto, ya no solo existía un problema de racismo epistémico, sino un epistemicidio que dio lugar a un texto sin sujeto al prescindir de los nombres y de las voces que habían sido la condición de posibilidad de la experiencia que se estaba narrando.

Los fenómenos sociales son en sí mismos una producción de sentido que no son sostenibles sin su relación ontológica dado que estos consisten en acciones y representaciones llevadas a cabo por los sujetos. Esta omisión, tan común y tan paradójica en las investigaciones en el ámbito de las ciencias sociales, simplemente negaba la posibilidad de la emergencia de "otro conocimiento" y de la ecología de saberes señalada previamente en el marco teórico del texto que pudiera confrontar la monocultura del conocimiento científico. En el caso de la investigación en el ámbito de las migraciones, estas omisiones son, en parte, fruto del legado de los procesos de una forma de etnificación a los que han sido sometidos los sujetos migrantes y que han reproducido la imagen de un sujeto aún "en minoría de edad" por quien debía de hablarse y del cual no se esperaba que tuviera capacidad de raciocinio propio.

Con respecto al proceso seguido, el hecho de no haber informado detalladamente de la fase de escritura que se estaba llevando a cabo y la posterior devolución de un texto cerrado, listo para poder enviarse, fue interpretado como un "hablar por ellas" o "hablar de ellas" sin el debido consentimiento ${ }^{33}$. Siguiendo a Rita Segato ${ }^{34}$, estas mujeres no aceptaron un texto que sustituía el significado por el acto mismo de significar. Su exclusión del proceso de escritura en el que se reemplazaba sus voces, contingentes y enfrentadas, por un relato académico, racional y unitario, las había subalternizado e incluso deslegitimizado, excluyéndolas de su propia experiencia. En esta línea, Chakrabarty $^{35}$ también nos advierte que la historia siempre es el resultado del encuentro de muchas narrativas enfrentadas, sin embargo, los procesos de investigación no recogen esta pluralidad debido a que en ocasiones se investiga para validar hipótesis en vez de hacernos cargo de la complejidad y versatilidad de la realidad social. Es por esto que los investigadores terminan sacrificando la sustancia poética de los actos que observan ${ }^{36}$ y no se llega a cuestionar el acto de violencia simbólica que se ejerce al reducir "experiencias con sentido" a meros datos que posteriormente serán transformados en explicaciones

\footnotetext{
${ }^{33}$ Debemos recordar en este punto, que el consentimiento dado para la escritura del texto, no necesariamente implica la validación del contenido, aunque en una gran mayoría casos, este consentimiento sea aplicado tanto al proceso como al producto.

34 SEGATO, op. cit., p. 35.

${ }^{35}$ CHAKRABARTY, op. cit., p. 88.

36 SEGATO, op. cit., p. 38.
} 
válidas, que no validadas, sobre determinados sujetos y su realidad social. Esta violencia simbólica es raramente puesta de manifiesto y los mecanismos que los investigadores podrían emplear para aminorarla son todavía un procedimiento secundario en el área de la investigación en migraciones.

En el caso presentado, esta violencia se agravó por las posibilidades que los investigadores disponen para seguir adelante con sus intereses, en ocasiones desconectados abismalmente de los intereses de las personas migrantes, y por la supuesta labor de producción de equivalencias entre dos mundos, el de los migrantes y el de los investigadores, que se intentó llevar a cabo sin reparar en las distorsiones que entraña la simplificación de las "experiencias con sentido".

Para ir concluyendo este apartado, señalaremos que para el comité de ética ${ }^{37}$ que revisó el texto y el comité científico del simposio al que fue presentado, las incoherencias en el marco teórico antes mencionadas y todos los detalles hasta ahora descritos pasaron inadvertidos. Este hecho pone de relieve dos realidades. Por un lado, podríamos señalar que no llama la atención un texto que narra una experiencia "sin sujetos", en tanto que esta es la norma y no la desviación. Por otro lado, se puede deducir que los presupuestos éticos frente a los que se examinan las praxis llevadas a cabo durante las investigaciones, responden a una ética de mínimos cuestionable en sí misma y que, en la mayoría de los casos, se corresponden con unos principios generalistas que no permiten examinar los dilemas y problemas de cada área de conocimiento. La forma en la que se realiza la investigación en el ámbito de los estudios migratorios deja poco lugar para examinar las apropiaciones, intencionadas o desintencionadas, que los investigadores realizan sobre el conocimiento producido por los sujetos migrantes. De igual forma, tampoco se indaga en la posibilidad de realizar investigaciones falsables, no dogmáticas, dado que la manera en la que están siendo producidas lo impide. Estas circunstancias nos remiten a las dificultades que existen para examinar la viabilidad ética de la investigación en migraciones y al hecho de que aún se carece de un corpus de principios y presupuestos éticos que permita a los investigadores tomar conciencia de los problemas de carácter ético inherentes a su propio trabajo.

${ }^{37}$ Debemos señalar que los comités de ética en ciencias sociales, de muy reciente creación, trayectoria e implantación en centros de investigación y universidades, son aún fuente de controversia y conflicto en tanto que estos se entienden como un atentado a la autonomía de los investigadores y como un mecanismo de control y censura en la investigación. Por otro lado, estos comités surgen ligados a agencias financiadoras y centran buena parte de su actividad en cuestiones meramente burocráticas que se alejan de los intereses de los investigadores. El debate sobre su pertinencia y sobre el alcance de sus funciones es una cuestión abierta que debería abordarse con el fin de redundar en el mejor desarrollo de todos los aspectos práctica investigadora desde el punto de vista ético. Sobre estas cuestiones ver: ACHÍO TACSAN, Mayra. Los comités de ética y la investigación en Ciencias Sociales; DINGWALL, Robert. The ethical case against ethical regulation in humanities and social science research, Twenty-First Century Society; ROVALETTI, M. Lucrecia. La evaluación ética en las ciencias humanas y/o sociales. La investigación científica: entre la libertad y la responsabilidad. 


\section{Reflexiones finales}

A la luz de lo que se ha ido exponiendo en este texto, más que conclusiones al uso, podrían esbozarse una serie de reflexiones finales sobre los aspectos éticos surgidos durante el proceso de investigación en este estudio de caso con personas migrantes. Retomando la idea que señalábamos al inicio de este texto, debemos insistir en que la investigación y la producción de conocimiento son actos situados, no neutrales, que no pueden entenderse y desarrollarse desligados de su condición ética. Es por esto que los procesos de investigación y el conocimiento resultante de ellos, deben concebirse como el resultado de una construcción compartida "con" y como un proceso dialéctico en la que los distintos participantes contribuyen, desde distintos roles y de modo equivalente a la producción de conocimiento. Esta forma de entender los procesos de investigación debe tener consecuencias visibles en la forma en la que se desarrollan las relaciones en el trabajo de campo, el proceso de escritura y la posterior difusión y devolución de los trabajos. La revisión rigurosa de los presupuestos ontológicos y epistemológicos de los cuales se parte en una investigación, así como de su desarrollo metodológico, debería constituir un paso previo y central en los procesos de investigación. Igualmente, deberían examinarse aquellas categorías analíticas y conceptuales mediante las cuales se trata de aprehender la realidad y de representar a quienes la conforman, debido a que muchas de las categorías empleadas en el ámbito de las migraciones, y en concreto para describir a los sujetos migrantes, son errónea o injustificadamente usadas. Estas categorías, generalmente producidas desde un contexto específico y tributarias de una tradición de pensamiento occidentalocéntrico, pretenden ser universalmente válidas para dar cuenta de la diversidad humana y de una gran variedad de procesos sociales, aunque las taxonomías y clasificaciones que de ellas se derivan terminan generando procesos de diferenciación, especialmente problemáticos cuando la diversidad es atribuida principalmente a los movimientos migratorios.

Una mayor vigilancia epistemológica evitaría, por un lado, la constante instrumentalización de los sujetos migrantes como proveedores de datos útiles para los investigadores y, por otro, la producción de conocimiento desde alteridades invisibilizadas, silenciadas o suprimidas ${ }^{38}$. Igualmente, esta vigilancia permitiría identificar una serie de prácticas, no necesariamente carentes de ética, destinadas a adecuar la necesaria entropía de los procesos de investigación a las reglas de producción de conocimiento científico, mediante las cuales se deslegitiman y se descartan "otros" saberes etnificados. Frente a los actos de violencia epistémica, y dadas las limitaciones que los científicos

38 TAVARES, Manuel. A Filosofia Andina 1: Uma interpelação ao pensamento ocidental. Colonialismo, colonialidade e descolonização para uma interdiversidade de saberes (J. Estermann), p. 213. 
sociales enfrentan en los procesos de investigación, deberíamos asumir y hacer evidente la imposibilidad de la horizontalidad del trabajo analítico, aunque esto no implica que se desatiendan los principios de buena praxis que deben guiar cualquier investigación. La devolución del producto final, si es que se llega a realizar, sirve para tranquilizar las conciencias de muchos investigadores tras un periodo de asedio y extracción a un determinado grupo o comunidad. Esta devolución y la posterior inclusión de las observaciones que se realicen, no es concebida como un acto ético necesario en el proceso de investigación que puede contribuir sustancialmente a la mejora de la producción de conocimiento.

Con respecto a las motivaciones que justificaron la difusión anonimizada del trabajo, y que apelaban al interés general que hubiera podido tener el texto, lo que en realidad se estaba tratando de poner de manifiesto es la posibilidad que un bien de carácter finalista puede tener para justificar los medios necesarios para conseguir un determinado beneficio. Los principios finalistas aplicados a la investigación deberían examinarse con gran detenimiento dado que en muchas ocasiones se confunde la utilidad con el valor social de las investigaciones, y en este caso concreto, el texto no cumplía ninguna de estas dos condiciones.

Finalmente, señalaremos que la condición de hecho social total que poseen las migraciones, hace especialmente complejo generar un corpus de principios éticos que pueda orientar la práctica investigadora, pero el análisis de esta experiencia ha puesto de manifiesto la posibilidad que ofrece el estudio de experiencias concretas para ir avanzando en esta necesidad.

\section{Bibliografía}

ACHÍO TACSAN, Mayra. Los comités de ética y la investigación en Ciencias Sociales. Revista de Ciencias Sociales (Cr), n. 99, 2003, p. 85-95.

APARICIO GÓMEZ, Rosa; TORNOS CUBILLOS, Andrés. Las asociaciones de inmigrantes en España. Una visión de conjunto. Documentos del Observatorio Permanente de la Inmigración. Madrid: Ministerio de Trabajo e Inmigración, 2010. ARANGO VILA-BELDA, Joaquín. Inmigración y diversidad humana. Una nueva era en las migraciones internacionales. Revista de Occidente, n. 268, 2003, p. 5-21.

BECKER, Howard; GEER, Blanche. Participant observation: the analysis of qualitative field data. In BURGESS, Robin G. (ed.). Field research: a sourcebook and field manual. London: Allen \& Unwin, 1982, p. 239-250.

BERGER, Peter; LUCKMANN, Thomas. La construcción social de la realidad. Buenos Aires: Amorrortu Editores, 2003.

BRUBAKER, Roger; COOPER, Frederik. Beyond "identity". Theory and society, v. 29, 2000, p. 1-47.

BRUBAKER, Roger. Ethnicity without groups. Archives Européennes de Sociologie, v. XLIII, n. 2, 2002, p. 163-189. 
CHAKRABARTY, Dipesh. Historias de las minorías, pasados subalternos. Historia y grafía, n. 12, 1999, p. 87-111.

DINGWALL, Robert. The ethical case against ethical regulation in humanities and social science research. Twenty-First Century Society, v. 3, n. 1, 2008, p. 1-12.

GARCíA CASTAÑO, Francisco Javier; LÓPEZ FERNÁNDEZ, Rosalía; THAMM, Miriam. Sujetos y territorios en el estudio de las migraciones desde la antropología en España. QuAderns-e, v. 19, n. 2, 2014, p. 100-125.

GROSFOGUEL, Ramón. La descolonización del conocimiento: diálogo crítico entre la visión descolonial de Frantz Fanon y la sociología descolonial de Boaventura de Sousa Santos. In AA.VV. Formas-Otras: Saber, nombrar, narrar, hacer. Barcelona: CIDOB, 2011.

HALL, Stuart. El espectáculo del "Otro". In RESTREPO, Eduardo; WALSH, Catherine; VICH, Víctor (eds.). Sin garantías. Trayectorias y problemáticas en estudios culturales. Popayán: Universidad Javeriana-Instituto de Estudios Peruanos-Universidad Andina Simón Bolívar-Envión, 2010, p. 419-445.

KAWULICH, Barbara. Participant Observation as a Data Collection Method. Forum Qualitative Sozialforschung/Forum: Qualitative Social Research, v. 6, n. 2, Art. 43, 2005.

LÓPEZ FERNÁNDEZ, Rosalía. Luchas y aprendizajes desde espacios subalternos en el contexto asociativo. Procesos de empoderamiento y participación de mujeres migrantes empleadas de hogar. Texto aceptado para Simposio "Aproximación crítica a la participación y el empoderamiento. Estudios sobre minorías socioculturales y grupos sociales marginalizados" del XIV Congreso de Antropología. Antropologías en trasformación: Sentidos, compromisos y utopías. Valencia, España, septiembre 2017.

MÁRQUEZ FERNÁNDEZ, Álvaro. La ética del investigador frente a la producción y difusión del conocimiento científico. Revista Venezolana de Gerencia, v. 6, n. 16, 2001, p. 632-650.

McNAY, Lois. Gender and Agency: Reconfiguring the Subject in Feminist and Social Theory. London: Blackwell, 2000.

MESÍA MARAVÍ, Rubén. Contexto ético de la investigación social. Investigación Educativa, v. 11 n. 19, 2007, p. 137-151.

OLMOS ALCARAZ, Antonia. La población inmigrante extranjera y la construcción de la diferencia. Discursos de alteridad en el sistema educativo andaluz. Tesis leída en la Universidad de Granada, 2009.

PATEMAN, Carole. The sexual contract. Standford: Standford University Press, 1988. PRADA, Miguel Ángel de; ACTIS, Walter; PEREDA, Carlos. ¿Cómo abordar el estudio de las migraciones? Propuesta teórico metodológica. In CHECA, Francisco (coord.). Las migraciones a debate. De las teorías a las prácticas sociales. Barcelona: Icaria, 2002, p. 17-54.

ROVALETTI, M. Lucrecia. La evaluación ética en las ciencias humanas y/o sociales. La investigación científica: entre la libertad y la responsabilidad. Acta Bioethica, v. 12 , n. 2, 2006, p. 243-250. 
SANTAMARÍA LOZANO, Enrique. Inmigración y barbarie. La construcción social y política del inmigrante como amenaza. Papers: Revista de Sociología, n. 66, 2002, p. 59-75.

SANTAMARÍA LOZANO, Enrique. La incógnita del extraño. Una aproximación a la significación sociológica de la "inmigración comunitaria". Barcelona: Anthropos, 2002.

SASSEN, Saskia. Contrageografías de la globalización. Género y ciudadanía en los circuitos transfronterizos. Madrid: Traficantes de sueños, 2003.

SCHEFFLER, Ted. The ideology of binary opposition: Subject/object Duality and Anthropology. Dialectical Anthropology, v. 6, n. 2, 1981, p. 165-169.

SEGATO, Rita Laura. Una paradoja del relativismo. El discurso racional de la antropología frente a lo sagrado. In RUFER, Mario; GORBACH, Frida (eds.). (In) disciplinar la investigación. Archivo, trabajo de campo y escritura. México: Siglo XXI-UAM, 2016.

SOUSA SANTOS, Boaventura de. La Sociología de las Ausencias y la Sociología de las Emergencias: para una ecología de saberes. In IDEM. Renovar la teoría crítica y reinventar la emancipación social (encuentros en Buenos Aires). Buenos Aires: CLACSO, 2006.

SOUSA SANTOS, Boaventura de. Decolonizar el saber, reinventar el poder. Montevideo: Ediciones Tricicle, 2010.

SOUSA SANTOS, Boaventura de. Epistemologías del Sur. Utopía y Praxis Latinoamericana, n. 54, 2011, p. 17-39.

TAVARES, Manuel. A Filosofia Andina 1: Uma interpelação ao pensamento ocidental. Colonialismo, colonialidade e descolonização para uma interdiversidade de saberes (J. Estermann). EccoS Revista Científica, n. 32, 2013, p. 197-252.

VASILACHIS DE GIALDINO, Irene. Pobres, pobreza, identidad y representaciones sociales. Barcelona: Gedisa, 2003.

VIDAL FERNÁNDEZ, Fernando. Pan y rosas. Fundamentos de exclusión social y empoderamiento. Madrid: Fundación FOESSA, 2009.

Recibido para publicación en 28.05.2017

Aceptado para publicación en 27.06.2017

Received for publication in May 28 ${ }^{\text {th }}, 2017$

Accepted for publication in June $27^{\text {th }}, 2017$

ISSN impresso 1980-8585

ISSN eletrônico 2237-9843

http://dx.doi.org/10.1590/1980-85852503880005006 